\title{
Revision of SKED: Needs and desires
}

\author{
MARK F. LEWIS \\ Civil Aeromedical Institute, FAA \\ Oklahoma City. Oklahoma 73125 \\ and \\ TIMOTHY F. ELSMORE \\ Walter Reed Army Institute of Research \\ Washington, D.C. 20012
}

\section{Modifications are suggested for Snapper's SKED system.}

Snapper's SKED system (Snapper \& Walker, 1971) for computer control of operant behavior chambers has been in use in our laboratories for several months. The system has many shortcomings. To a large extent, these shortcomings are related to the historical development of the SKED system, which was intended as a low-cost computer system to replace conventional process control and data acquisition systems in the operant laboratory (Snapper \& Kadden, 1972). The system was designed to operate with a PDP-8 computer with $4 \mathrm{~K}$ of memory, a Teletype, and a simple interface system, and, in fact, it works well with this hardware configuration. SKED itself is designed to handle 10 chambers simultaneously, with up to 12 inputs and 12 outputs per station. However, with its current limitation to $4 \mathrm{~K}$ of memory, only very simple experiments can be run if all chambers are implemented. Increases in the complexity of experiments to be run decrease the number of chambers that can be operative at any given time. The restriction to paper-tape $1 / O$ also arises from the cost-conscious background of the SKED system. These difficulties with the system can be solved by modifying the SKED system such that it can operate in more advanced hardware configurations.

A step in this direction has been taken by Lewis and Savage (1972), who have experimented with a revised version of SKED which uses the device-independent features of the OS/8 operating system for compiling, loading, and running SKED programs. This modified SKED dumps data in OS/ 8 files that are suitable for analysis by OS/8 FOCAL or FORTRAN. Their compiler currently runs in $12 \mathrm{~K}$ of memory, but offers no new features other than the device independence of OS $/ 8$. Their run-time system (RTS) requires $8 \mathrm{~K}$, but yields substantially greater space for SKED programs than does the $4 \mathrm{~K}$ paper-tape version. A significant disadvantage of the Lewis and Savage (1972) RTS is that all programs to be run must be loaded, started, and dumped simultaneously. This arises from the fact that OS $/ 8$ device handlers are not interrupt driven. Thus, if experimental sessions in different chambers differ significantly in length. considerable dead time will be experienced in some chambers. Thus, the Lewis and Savage version of SKED offers both advantages and disadvantages.

\section{SUGGESTED MODIFICATIONS TO SKED}

These as well as other deficiencies in the SKED system constitute significant constraints in the choice of experimental procedures. The following are our proposed modifications to SKED that would help to alleviate these problems.

Perhaps most importantly, the SKED system should be restructured to allow state tables to be loaded into any memory field. This would require an additional status word for each chamber to serve as a field pointer, and restructuring of the SKED subroutines to permit them to be called from any memory field. Indeed, this has been done in portions of the Lewis and Savage SKED. The compiler would also require modification such that each state table contained a field pointer.

A less serious difficulty is the restriction to 12 stimulus outputs per chamber, imposing additional constraints on experimental design. Complex stimulus control experiments, in particular, may require more stimuli. This could be solved by allocation of three stimulus status words per chamber rather than one. This would allow up to 36 stimuli per box.

As currently designed, SKED programming is greatly simplified by the use of three assignment functions. The F1 function allows incrementing of variables, the F2 function allows the assignment of specific values to a variable, and the F3 function allows the user to call Assembly language subroutines to perform special-purpose functions. It seems clear that the addition of two new assignment functions would simplify programming even further. As currently configured, SKED does not permit generation of random numbers. Thus, procedures such as random ratio schedules and discrimination procedures requiring randomization of trial sequences are difficult if not impossible to implement. Further, sequences of trials in trial-by-trial procedures or intervals or ratios in variable-interval or variable-ratio schedules that are of any length use a great deal of memory, requiring at least 12 memory locations for each value in the sequence. We propose two new functions that would handle these difficulties: An F4 function would assign values to variables by performing a sequential table look-up. and an F5 function would operate similarly, but would use a pseudorandom number generator to select tabled values. These two functions permit the user to program variable-interval or -ratio schedules using any predetermined sequence of intervals (or ratios) without requiring a different state for each interval or ratio value. 


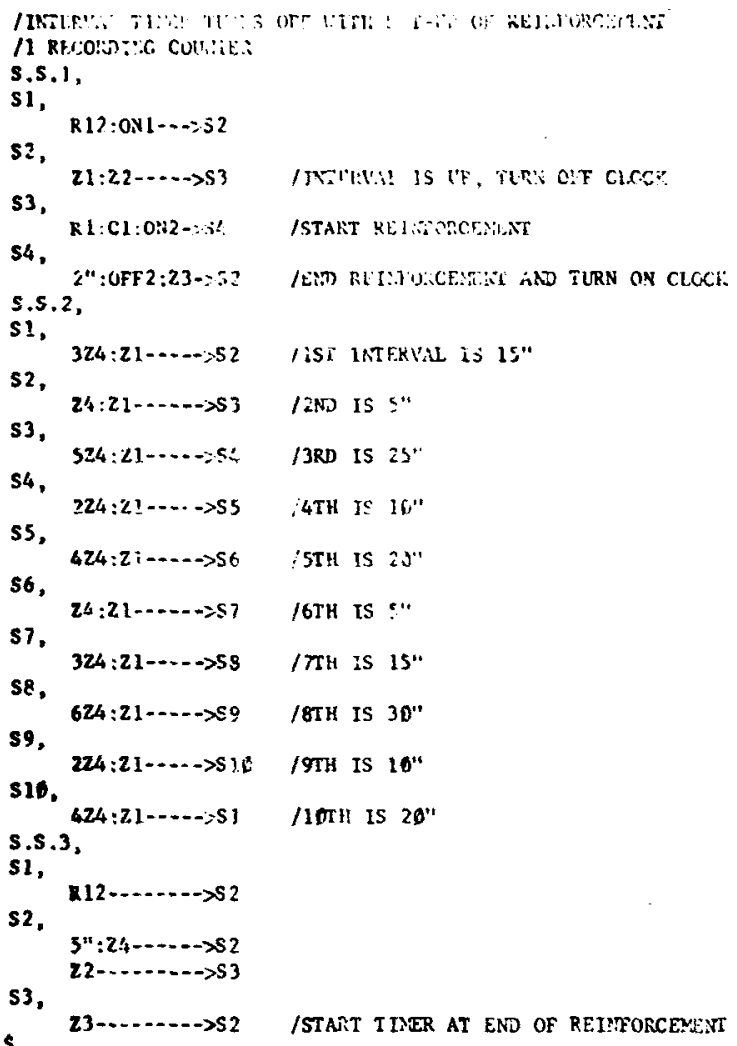

$\$$

Fig. 1. Ten-interval variable-interval schedule using current SKED language.

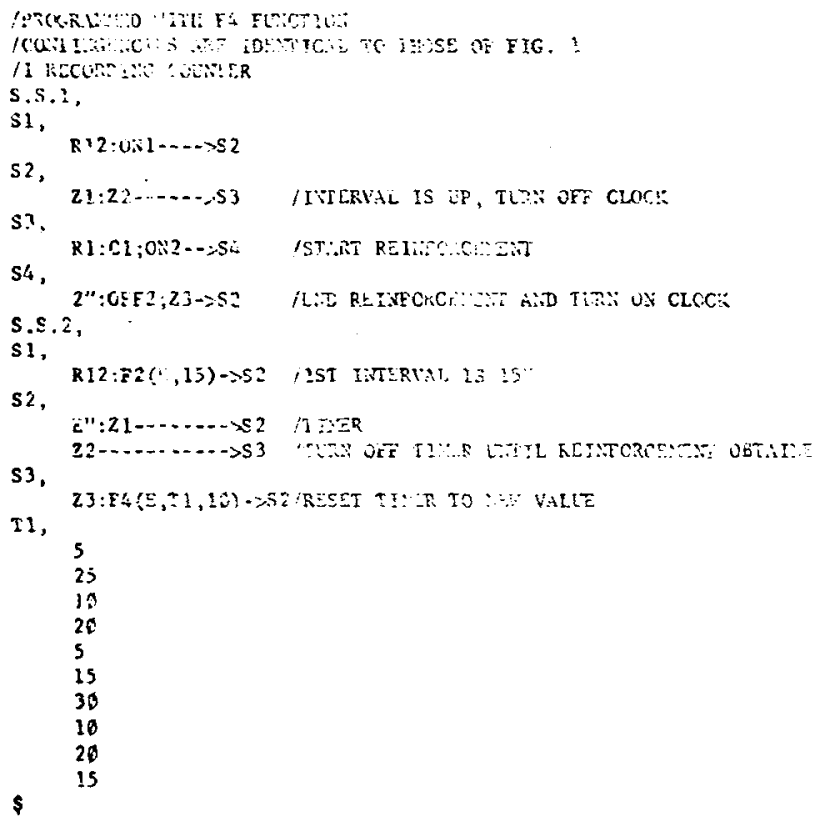

Fig. 2. Ten-interval variable-interval schedule using proposed F4 function. thus saving memory space and simplifying the writing of programs. These functions would similarly simplify programming of trial-by-trial procedures. Figure 1 shows an example of a 10 -interval variable-interval schedule as programmed by the current SKED system. The inefficiency of requiring separate states for each of the 10 different intervals is obvious. Figure 2 shows how use of our proposed $\mathrm{F} 4$ function simplifies this program and any other program requiring a predetermined sequence of values for a variable. Note the use of "T 1 " to define a table. The F4 function requires three arguments: the variable to be assigned ( $E$ in the example), the table to be used ( $\mathrm{T} 1$ in the example), and a numerical argument indicating the last tabled entry to be used (10 in the example). Obviously, this value cannot be larger than the number of entries in the table, but allows use of less than the complete number of entries to be used. In Fig. 2, the $F 4$ function assigns values to $E$, beginning with the first entry in Table $T 1$ and ending with the 10 th, after which it returns to the start of the table. If the $\mathrm{F} 5$ function were used rather than the F4 function, a variable-interval schedule would be generated in which successive interval values would be randomly chosen from $T 1$, rather than chosen sequentially. The arguments, with the exception of the assignment function number, would be identical.

An additional difficulty with the SKED system is that it does not permit simultaneous compilation of new programs while the RTS is running. In some experimental environments, such as those requiring 24-h monitoring of behavior, such a foreground-background arrangement is highly desirable, since new experiments could then be compiled without disrupting those currently in progress. In an OS/8 environment, such an arrangement would be impossible to achieve because of the lack of interrupt-driven device handlers. This suggests that some other operating system would be more appropriate for SKED users.

The authors invite other SKED users to suggest additional features that could profitably be incorporated into a new version of SKED that would add to the convenience and utility of the system. We would particularly welcome suggestions regarding the choice of an operating system that would be more suitable than $\mathrm{OS} / 8$. We are actively working on most of the revisions we have indicated. and welcome any help we can get.

\section{REFERENCES}

Lewis, $M, F$ \& Savage, $P$ An OS/8 SKED software system. In Proceedings of the Fall 1972 DECUS Symposium, 1972.

Snapper, A. G., \& Kadden, R. M. Time-sharing in a small computer based on use of a behavioral notation system. In $B$. Weiss (Ed.), Digital computers in the behavior laboratory. New York: Appleton-Century-Crofts. 1972.

Snapper, A. G., \& Walker, A. The SKED software system. DECUS Program Library. DECUS 8-465. 1971 